\title{
Galanin Administration Partially Restores Erectile Function After Cavernous Nerve Injury and Mediates Endogenous Nitrergic Nerve Outgrowth In Vitro
}

Emmanuel Weyne, MD, Johanna L. Hannan, $\mathrm{PhD}^{2,3}$ Thomas Gevaert, MD, $\mathrm{PhD}^{4}{ }^{4}$ Yodi Soebadi, MD, Hotaka Matsui, MD, 2,5 Fabio Castiglione, MD, ${ }^{1,6}$ Koenraad van Renterghem, MD, PhD, ${ }^{7}$ Dirk De Ridder, MD, PhD, Frank Van der $\mathrm{Aa}, \mathrm{MD}, \mathrm{PhD}^{1}$ Trinity J. Bivalacqua, $\mathrm{MD}, \mathrm{PhD}^{2}$ and Maarten Albersen, $\mathrm{MD}, \mathrm{PhD}^{\top}$

\begin{abstract}
Background: Previously, we found that the neuropeptide galanin was strongly upregulated soon after bilateral cavernous nerve injury (BCNI) and that galanin and its receptors were expressed in nitrergic erectile innervation. Galanin has been observed to exert neuroregenerative effects in dorsal root ganglion neurons, but evidence for these effects in the major pelvic ganglion (MPG) after BCNI is lacking.
\end{abstract}

Aim: To evaluate the neurotropic effects of galanin receptor agonists and antagonists in vitro in nitrergic neurons and MPG and in vivo in rats after BCNI.

Methods: Male Sprague-Dawley rats underwent BCNI and sham surgery. Organ culture and single-cell neuron culture of the MPG were performed. Osmotic pump treatment with the galanin agonist in vivo and measurement of erectile response to electrostimulation after BCNI, immunohistochemical localization of galanin and receptors in the human neurovascular bundle, and myographic analysis of rat corpus cavernosum smooth muscle relaxation to galanin receptor agonists were investigated.

Outcomes: Neurite outgrowth in vitro and erectile response to electrostimulation after BCNI in vivo, immunohistochemical localization of galanin and receptors, and penile muscle relaxation in vitro.

Results: Galanin showed neurotrophic action in vitro and inhibition of endogenous galanin significantly impaired neurite outgrowth in nitrergic but not in sympathetic MPG neurons. In vivo administration of a selective galanin receptor-2 agonist, M1145, resulted in partial recovery of erectile function (EF) after BCNI. Galanin did not act as a direct vasodilator on corpus cavernosum muscle strips.

Clinical Translation: Endogenous neurotrophins such as galanin could be used as a strategy to improve EF for patients after BCNI from radical prostatectomy.

Strengths and Limitations: We evaluated the effect of galanin on nerve regeneration and EF recovery in vivo and in vitro. Limitations include the lack of washout period for the in vivo experiment and absence of differences in the expression of neuronal markers between treatment groups.

Conclusions: We identified galanin as a potential endogenous mechanism for nerve regeneration after BCNI, which could play a physiologic role in EF recovery after radical prostatectomy. In vivo treatment with exogenous galanin was beneficial in enhancing EF recovery after BCNI, but further research is necessary to understand the underlying mechanisms. Weyne E, Hannan JL, Gevaert T, et al. Galanin Administration Partially Restores Erectile Function After Cavernous Nerve Injury and Mediates Endogenous Nitrergic Nerve Outgrowth In Vitro. J Sex Med 2018;XX:XXX-XXX.

Copyright @ 2018, International Society for Sexual Medicine. Published by Elsevier Inc. All rights reserved.

Key Words: Galanin; Erectile Dysfunction; Radical Prostatectomy; Neuroregeneration; Nerve Culture; Cavernous Nerve Injury

Received December 16, 2017. Accepted February 4, 2018.

'Department of Urology, KU Leuven and University Hospitals, Leuven, Belgium;

${ }^{2}$ Brady Urological Institute, Johns Hopkins, Baltimore, MD, USA;

${ }^{3}$ Department of Pathology, KU Leuven and University Hospitals, Leuven, Belgium;

${ }^{4}$ Department of Physiology, East Carolina University, Greenville, NC, USA;

\author{
${ }^{5}$ Department of Urology, University of Tokyo, Tokyo, Japan; \\ שUniversity College of London, London, UK; \\ ${ }^{7}$ Department of Urology, Jessa Hospital, Hasselt, Belgium \\ Copyright ( $)$ 2018, International Society for Sexual Medicine. Published by \\ Elsevier Inc. All rights reserved. \\ https://doi.org/10.1016/j.jsxm.2018.02.013
}




\section{INTRODUCTION}

Erectile dysfunction (ED) remains a common sequela of radical prostatectomy (RP). Currently, younger and healthier patients are diagnosed with localized prostate cancer and have high expectations of regaining erectile function (EF) after surgery. ${ }^{1}$ Despite the refinement of nerve-sparing techniques and technical advancement with the introduction of robotic surgery, ED ranges from $14 \%$ to $69 \%$ after RP. ${ }^{2}$ ED occurs as a direct consequence of damage by traction, compression, and use of electrocautery in the neighborhood of cavernous nerves $(\mathrm{CNs})$ located on the dorsolateral aspect of the prostate. This nerve injury will initiate a neurodegenerative cascade called Wallerian degeneration, resulting in temporary denervation of the corpus cavernosum. This denervation eventually leads to fibrosis and loss of cavernosal smooth muscle mass, leaving the patient with a severe form of ED that is difficult to treat. ${ }^{3}$

Currently no causal therapies exist to restore EF after RP. Current therapies, such as phosphodiesterase type 5 inhibitors, attempt to restore relaxation of the end organ, and penile rehabilitation with these agents have not shown conclusive results toward EF preservation and recovery. ${ }^{4}$ The autonomic nervous system has a limited capacity to regenerate after nerve injury, which is mediated by the endogenous release of neurotropic factors stimulating the survival and outgrowth of neuronal cells. ${ }^{5}$ It is of utmost importance to better understand the mechanism and neurotrophic factors that are involved in $\mathrm{CN}$ regeneration to find new treatments that can truly maximize EF recovery after RP. The exogenous administration of neurotrophic and growth factors in preclinical rat models has resulted in increased EF recovery after $\mathrm{CN}$ injury $(\mathrm{CNI}){ }^{1}$

In a previous study, we found that expression of the neurotrophin galanin was 180 -fold upregulated in the major pelvic ganglion (MPG) of rats in the early phase after CNI. Furthermore, galanin and its 3 known receptors were located in neuronal nitric oxide synthase (nNOS)-positive neurons of the MPG, providing erectile innervation to the penis. ${ }^{6}$ Galanin has 3 known G-protein-coupled receptors that are abundantly expressed in the central nervous system and dorsal root ganglion (DRG) of sensory neurons. ${ }^{7,8}$ Galanin was found to act as a neurotrophic factor in peripheral nerves because galanin knockout mice showed increased long-term sensory and motor deficits after nerve injury that could be salvaged by the addition of galanin. ${ }^{9}$ Neuroregenerative action of galanin is mediated by galanin receptor-2 (GalR2), which stimulates small GTPases to increase growth cone dynamics and neurite outgrowth in regenerating nerves. ${ }^{10}$ The role of galanin and its neurotrophic potential in autonomic nerves such as the $\mathrm{CN}$ has not been investigated.

In this study, we investigated the neurotropic potential of galanin in CNI. In the 1st step, a whole mount culture of the MPG was used to evaluate the neurotrophic action of galanin in vitro. A dissociated nerve culture was used to investigate the in vitro differential effect on growth of sympathetic vs parasympathetic nerves. In a 2nd step, we evaluated whether systemic galanin administration through an osmotic pump could improve EF recovery after CNI in vivo. As a 3rd step, the translational potential of galanin and its receptors was evaluated by performing immunohistochemical analysis on human neurovascular bundle specimens obtained after RP. Attached as supplementary data, we investigated whether galanin has a direct relaxation effect on human penile muscle strips in vitro.

\section{METHODS}

\section{Animals and Experimental Design}

Male Sprague-Dawley rats (Strain RjHan:SD, Janvier, Le Genest-Saint-Isle, France) 10 to 12 weeks old were used in this study. All experiments were approved by the animal ethics committee of the Ku Leuven (P169-2013) and Johns Hopkins (RA13M60). Rats were housed in standard laboratory conditions with a 12-hour light-dark cycle with access to food pellets and tap water ad libitum. Rats underwent sham or bilateral CNI (BCNI) surgery as described previously. ${ }^{6}$ Briefly, animals were anesthetized using $2 \%$ isoflurane, the prostate was exposed through a midline laparotomy, and the MPG and $\mathrm{CN}$ were identified bilaterally. In the BCNI groups, the $2 \mathrm{CNs}$ were injured by crushing with forceps 3 times for 15 seconds each time 2 to $3 \mathrm{~mm}$ distal to the MPG. ${ }^{5}$ Adequate crush was confirmed by an observable nerve decoloration, with the neurolemma remaining intact. In sham-operated animals, the $\mathrm{CN}$ was identified and the abdomen was closed. All BCNI surgeries were performed by the same surgeon.

\section{Pharmacologic Compounds}

For the different experiments, different galanin receptor agonists and antagonists were used (all from Tocris, IoTechne, Lille, France). M40 (non-selective) and M871 (selective for GalR2) served as antagonists. Rat galanin (non-selective) and M1145 (selective for GalR2) served as agonists. These compounds were dissolved and aliquoted in distilled water according to the manufacturer's instructions.

\section{Whole Mount Culture of MPG}

After sham or BCNI surgeries, MPGs were carefully dissected out at 48 hours after injury to be cultured in Matrigel $(n=4-5 /$ group). Whole MPGs were excised and embedded on reduced growth factor Matrigel in serum-free medium (RPMI-1640 with $1 \%$ penicillin and streptomycin; Gibco, Grand Island, NY, USA) as described previously. ${ }^{5}$ MPGs were covered with medium $1 \mathrm{~mL}$ with or without the addition of pharmacologic agents, which was changed every 24 hours and maintained at $37^{\circ} \mathrm{C}$ in a humidified atmosphere with $5 \% \mathrm{CO}_{2}$. Sham MPGs were treated with culture medium (vehicle), galanin $100 \mathrm{nmol} / \mathrm{L}$ (non-selective agonist), and M1145 $100 \mathrm{nmol} / \mathrm{L}$ (selective GalR2 agonist). 
Injured MPGs were treated with culture medium (vehicle), M40 $100 \mathrm{nmol} / \mathrm{L}$ (non-selective antagonist), and M871 $100 \mathrm{nmol} / \mathrm{L}$ (selective GalR2 antagonist; each $\mathrm{n}=4$ /group). Photographs of neurite growth at 48 and 72 hours were captured with a Nikon TE200 inverted microscope attached to a CCD camera, and digital images were analyzed in Elements software (Nikon Instruments, Melville, NY, USA). In each area of growth from the MPG, the 5 longest neurites were measured, as described previously. ${ }^{5,11}$ The averages of these measurements defined the neurite length for groups at each time point (20-25 neurites measured per MPG).

\section{Dissociated Nerve Culture}

Under isoflurane anesthesia, MPGs of non-nerve-injured rats were carefully dissected out and excised bilaterally under a dissection microscope. The ganglia were washed in $10 \%$ fetal calf serum and Neurobasal A Medium (basal medium; Invitrogen, Ghent, Belgium) and then incubated at $37^{\circ} \mathrm{C}$ in a mixture of collagenase $1 \mathrm{mg} / \mathrm{mL}$ (Gibco, Ghent, Belgium) and dispase 2.5 $\mathrm{mg} / \mathrm{mL}$ (Gibco) for 30 minutes. Digested ganglia were gently washed twice with basal medium and mechanically dissociated by mixing with syringes fitted with increasing needle gauges. Neurons were seeded on poly-L-ornithine and laminin-coated glass bottom chambers (Fluorodish WPI, Hertfordshire, UK) and cultured for 48 hours at $37^{\circ} \mathrm{C}$ in B27-supplemented Neurobasal A Medium containing $100 \mathrm{nmol} / \mathrm{L}$ of galanin, M871, or vehicle (neurobasal medium), which was changed every 24 hours ( $\mathrm{n}=6$ /group).

After 48 hours of culture, cells were fixed with buffered $4 \%$ paraformaldehyde for 30 minutes. Phosphate buffered saline, $0.3 \%$ Triton, $5 \%$ non-fat dry milk, and 5\% donkey serum were used for permeabilization and blocking. Rb anti-tyrosine hydroxylase (TH; 1:1,000; AB152, MilliporeSigma, Burlington, MA, USA) and Gt anti-nNOS (1:1,000; ab1376; Abcam, Cambridge, MA, USA) served as primary antibodies for incubation overnight on $4^{\circ} \mathrm{C}$. After washing with phosphate buffered saline plus Tween 20 for $3 \times 15$ minutes, 1:1,000 Alexa Fluor donkey anti-Rb 488 and anti-Gt 594 (Abcam) were applied for 1 hour at room temperature. After washing, glass bottoms were taken out of the well, plated, and mounted using Fluoroshield with 4',6diamidino-2-phenylindole (Sigma-Aldrich, Bornem, Belgium).

Photographs were captured with a microscope (Olympus, Tokyo, Japan) attached to a CCD camera and analyzed using Image 1.48 (National Institutes of Health, Bethesda, MD, USA). Differences in neurite outgrowth were analyzed between different neurons treated with vehicle vs M871 and vehicle vs galanin. For TH-stained neurons, images were set at a threshold using an automated function (Yen) and neurite surface area was measured. Immunofluorescent staining of the nucleus was removed to only include $\mathrm{TH}$-stained neurites. The nNOS staining included minimal background staining that did not allow automated analysis; therefore, the longest neurite per neuron was measured manually to compare outgrowth.

\section{Galanin Agonist Administration In Vivo}

Rats were divided in 3 groups ( $n=10 /$ group): (i) sham: identification of the $\mathrm{CN}$ without injury; (ii) vehicle: $\mathrm{BNCI}$ with implantation of an osmotic pump (Alzet 2ML4, Durect, Cupertino, CA, USA) containing vehicle solution $\left(\mathrm{H}_{2} \mathrm{O}\right)$; or (iii) M1145 group: active compound M1145 (selective galR2 agonist). The osmotic pump was placed subcutaneously to allow continuous administration at a rate of $2.5 \mu \mathrm{L} /$ hour or $3 \mu \mathrm{g} / \mathrm{kg} /$ day of M1145 during 4 weeks. The dosage of M1145 was derived and recalculated from a phase 3 clinical trial with subcutaneous administration of human recombinant ciliary neurotrophic factor. ${ }^{12} \mathrm{~A}$ pilot study was performed to assess the stability of the compounds dissolved in $\mathrm{H}_{2} \mathrm{O}$ at $37^{\circ} \mathrm{C}$ for up to 14 days using mass spectrometry. 4 weeks after surgery, erectile response to electrostimulation was evaluated as described previously at increasing voltages of $2.5,5$, and $7.5 \mathrm{~V}$ for 1 minute and erectile responses (ratio of intercavernosal pressure [ICP] to mean arterial pressure [MAP]) were measured. ${ }^{6}$ There was no washout period because this would have necessitated an additional surgery for pump explantation.

After erectile response measurements, animals were euthanized according to local regulations by cervical dislocation. Before euthanasia, plasma was derived from venous blood (vena cava) to detect the presence of M1145 in the blood using mass spectrometry in the M1145 and vehicle groups. The corpus cavernosum and MPGs were harvested to measure differences in expression of neuronal and smooth muscle markers using quantitative polymerase chain reaction. Differences in $\mathrm{nNOS}$, $\mathrm{TH}, \beta_{3}$-tubulin, and galanin expression were evaluated in the MPG and $\alpha$-smooth muscle actin, collagens I and III, nNOS, and $\mathrm{TH}$ were evaluated in the corpus cavernosum as described previously. ${ }^{6}$

\section{Localization Galanin and Receptors in Human Neurovascular Bundle}

Slides of human periprostatic neurovascular tissue were obtained from patients who underwent non-nerve-sparing RP $(n=4)$. Slides that contained a sufficient amount of nervous tissue were selected for immunostaining. The use of this tissue was approved by the ethical review board of the KU Leuven University and the patients provided fully informed written consent to the study.

Paraffin sections $(4 \mu \mathrm{m})$ were dewaxed in xylene and rehydrated with a graded ethanol series. Endogenous peroxidase activity was quenched by incubating slides with $0.5 \%$ hydrogen peroxide in methanol for 30 minutes. Slides were washed in Tris-buffered saline (TBS). For epitope retrieval, slides were placed in Tris-EDTA buffer $(\mathrm{pH}=9.0)$ for 30 minutes in a hot water bath at $98.5^{\circ} \mathrm{C}$. After washing in TBS, the slides were blocked for 60 minutes using $2 \%$ bovine serum albumin, $1 \%$ non-fat dry milk, and $0.1 \%$ Tween 80 in TBS (Sigma-Aldrich, St Louis, MO, USA), followed by incubation overnight at $4{ }^{\circ} \mathrm{C}$ in a humidified chamber with 1:100 $\mathrm{ms}$ anti-nNOS 
(Becton-Dickinson, Franklin Lakes, NJ, USA), 1:200 Rb antigalanin (Phoenix Pharmaceuticals, Burlingame, CA, USA), and 1:100 Rb anti-GalR1, 1:200 Rb anti-GalR2, and 1:100 Rb antiGalR3 (all from Alomone Labs, Jerusalem, Israel). Slides were rinsed in TBS, incubated with rabbit anti-mouse and swine antigoat biotinylated secondary antibody (Dako, Glosturp, Denmark) for 1 hour at room temperature, and incubated with a streptavidin biotin peroxidase complex (Roche Diagnostics, Mannheim, Germany). After the excess antibody had been washed out with TBS, the reaction product was developed with 3,3-diaminobenzidine substrate (10 minutes; Sigma-Aldrich), and the sections were counterstained with Mayer hematoxylin (Sigma-Aldrich). Human brain was stained as the positive control for all antibodies.

For immunofluorescent studies, an identical protocol was used, but instead immersion in 1:500 dilution of secondary antibody conjugated with Alexa-488 or Alexa-594 Fluor (Invitrogen, Carlsbad, CA, USA) in the dark was performed. Nuclear staining was performed with 4',6-diamidino-2-phenylindole $1: 10,000$ in phosphate buffered saline (D-3571; Invitrogen). Slides were mounted using Vectashield mounting medium (Vector Labs, Burlingame, CA, USA).

\section{Statistical Analysis}

Data are expressed as mean \pm standard error of the mean. Differences among multiple groups were compared by 1-way analysis of variance, followed by Tukey multiple-comparisons test (GraphPad Prism 5, GraphPad Software, San Diego, CA). A non-parametric Mann-Whitney U-test was used to compare the difference between 2 groups. A $P$ value less than .05 was considered statistically significant.

\section{RESULTS}

\section{Neurite Outgrowth in MPG Whole Mount Culture}

Mean neurite outgrowth of MPGs from sham-treated animals showed a trend toward increased outgrowth with selective GalR2 agonist treatment compared with vehicle treatment ( 48 hours, $445 \pm 56$ vs $355 \pm 30 \mu \mathrm{m} ; 72$ hours, $569 \pm 49$ vs 478 $\pm 50 \mu \mathrm{m} ; P>.05$; Figure $1 \mathrm{~B}, \mathrm{D})$. Inhibition of the galanin pathway in MPGs of injured rats with M871, a selective GalR2 antagonist, resulted in decreased neurite outgrowth compared with vehicle treatment (48 hours:, $452 \pm 19$ vs $632 \pm 32 \mu \mathrm{m}$, $P<.05 ; 72$ hours, $477 \pm 15$ vs $708 \pm 53 \mu \mathrm{m}, P<.01)$. Furthermore, decreased neurite outgrowth was more pronounced in M871-treated MPGs than non-specific galanin inhibition with M40 (48 hours, $452 \pm 19$ vs $607 \pm 43 \mu \mathrm{m}$, $P<.05$; Figure $1 \mathrm{C}, \mathrm{E})$.

\section{Neurite Outgrowth in Dissociated Cell Culture}

Pharmacologic stimulation with galanin did not significantly increase neurite outgrowth of nNOS-positive (Figure 2A2) and TH-positive (Figure 2B2) neurons obtained from non-nerveinjured rats compared with vehicle treatment. Inhibition of
A1

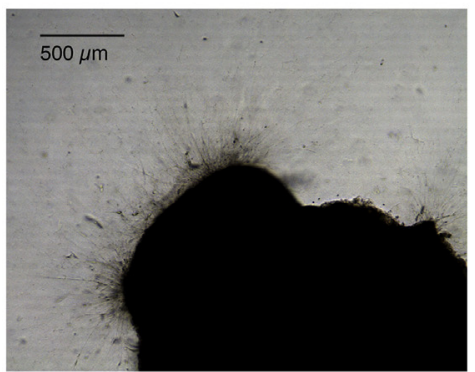

A2

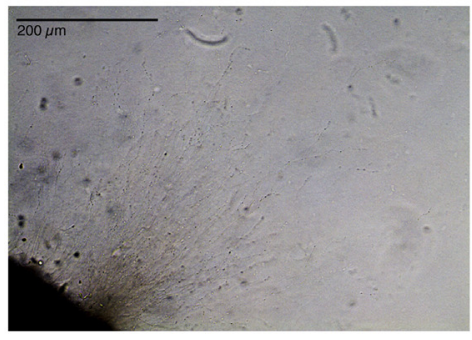

B

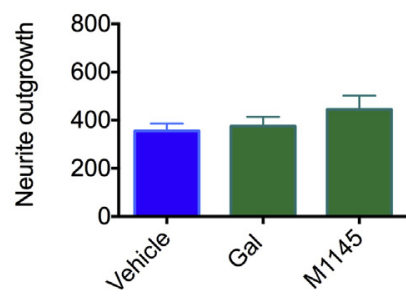

D

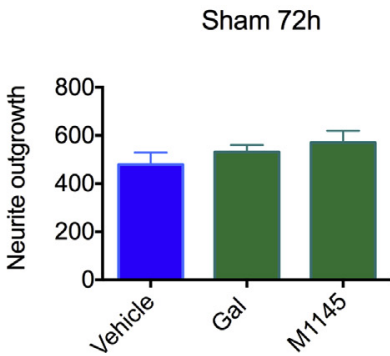

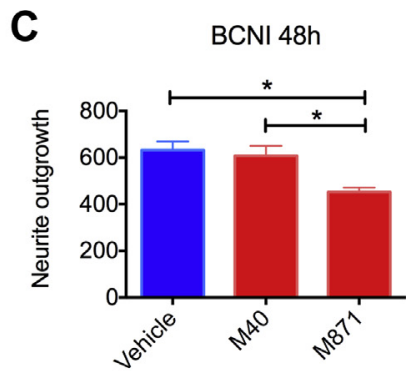

E

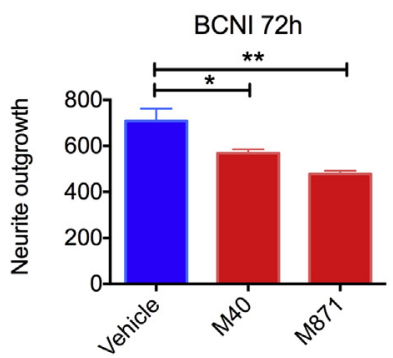

Figure 1. Panels $A 1$ and $A 2$ show a whole mount major pelvic ganglion cultured on a Matrigel-based layer with a distinctive zone of neurite sprouting. Panels B and D display neurite outgrowth of major pelvic ganglia obtained from sham animals at 48 and 72 hours, respectively, showing a non-significant trend toward increased neurite outgrowth with M1145, a selective galanin receptor-2 agonist. Panels $C$ and $E$ show galanin inhibition in major pelvic ganglia of injured rats resulting in impaired neurite outgrowth at 48 and 72 hours, respectively, in culture, which was more pronounced with $\mathrm{M} 871$, a selective galanin receptor-2 agonist. ${ }^{*} P<0.05 ;{ }^{* *} P<0.01$ ( $\mathrm{n}=4$ /group). $\mathrm{BCNI}=$ bilateral cavernous nerve injury. 
A1

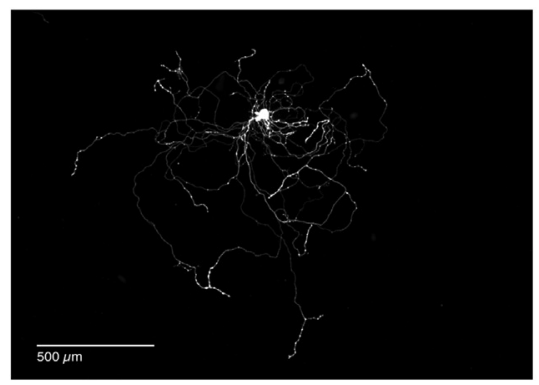

B1

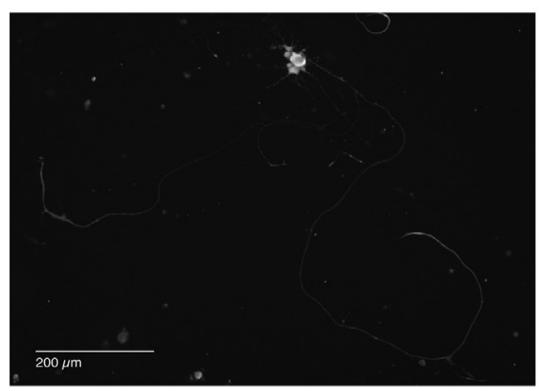

A2

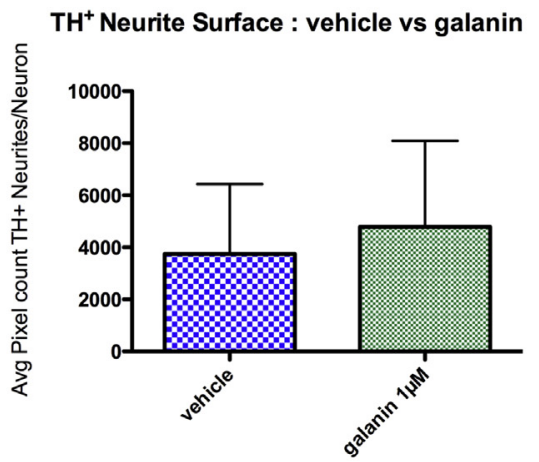

B2

nNOS ${ }^{+}$neurite length : vehicle vs galanin

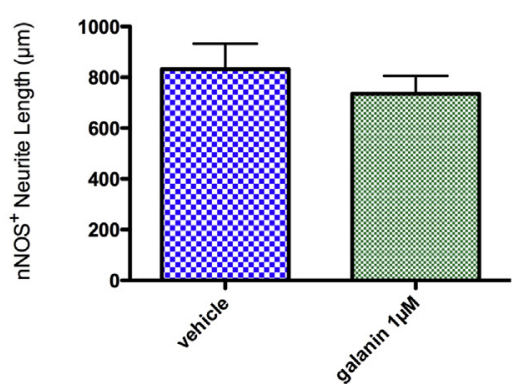

A3

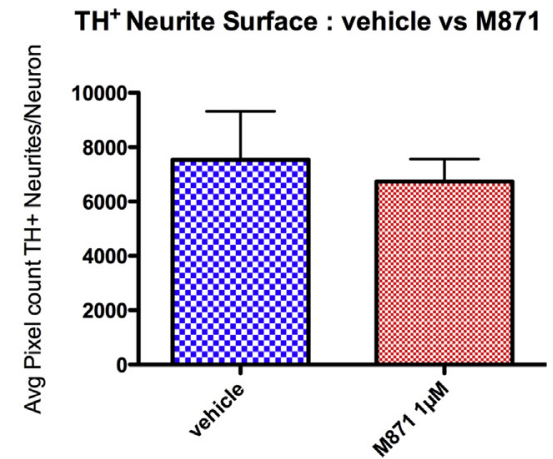

B3

nNOS $^{+}$neurite length : vehicle vs $\mathbf{M 8 7 1}$

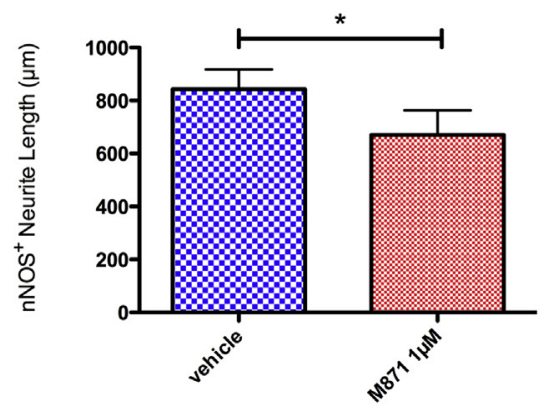

Figure 2. Galanin inhibition impairs neurite outgrowth of nitrergic pelvic neurons. Panels $\mathrm{B} 1$ and $\mathrm{A} 1$ show dissociated nerve culture of a sympathetic TH-positive neuron and a parasympathetic nNOS-positive neuron, respectively. Panels A2 and B2 show that pharmacologic stimulation with galanin did not significantly increase neurite outgrowth of nNOS-positive and TH-positive neurons, respectively, obtained from non-nerve-injured rats compared with vehicle treatment. Panels A3 and B3 show that inhibition of galanin signaling by M871, a specific galanin receptor- 2 agonist, resulted in significantly decreased neurite outgrowth of nitrergic but not of sympathetic neurons, respectively, compared with vehicle. ${ }^{*} P<0.05$ ( $n=6 /$ group). $\mathrm{nNOS}=$ neuronal nitric oxide synthase; $\mathrm{TH}=$ tyrosine hydroxylase.

galanin signaling by M871, a GalR2-specific antagonist, resulted in significant decreased neurite outgrowth of nitrergic neurons (843 \pm 34 vs $671 \pm 38 \mu \mathrm{m}, P<.05$; Figure 2 A3), but not of sympathetic neurons $(6,740 \pm 335$ vs 7,541 \pm 726 pixels/ neuron, $P>.05$; Figure $2 \mathrm{~B} 3)$ compared with vehicle.

\section{Osmotic Pump Administration of Galanin In Vivo and EF Recovery After BCNI}

EF was assessed by electrostimulation of the distal end of the $\mathrm{CN}$ at 28 days after surgery in all groups. As mentioned earlier, no washout protocol was performed because explantation of the osmotic pump would necessitate additional anesthesia and surgery. Stability of dissolved M1145 was confirmed with mass spectrometry for up to 14 days. The intensity of the detected compound remained stable, showing only minimal variance over time (Figure 3A). Blood serum samples obtained from M1145-treated rats showed a mass spectrometry spectrum and molecular weight consistent with M1145, which this was absent in vehicle-treated rats. The molecular weight of M1145 had shifted $1 \mathrm{Da}$ in vivo, which is consistent with deamidation from asparagine to aspartic acid at neutral pH (Figure 3B, C).
MAP did not differ between groups (data not shown). Sham-treated rats displayed normal ICP curves and ICP-toMAP ratios (Figure 4). In rats treated with vehicle, BCNI resulted in significantly impaired erectile responses to $\mathrm{CN}$ stimulation at all 3 voltages used for stimulation $(2.5 \mathrm{~V}, P<$ $.01 ; 5$ and $7.5 \mathrm{~V}, P<.001)$. In rats treated with $\mathrm{M} 1145$, partial recovery of erectile responses to electrostimulation was seen and these were significantly increased compared with vehicle-treated rats $(2.5 \mathrm{~V}, P<.05 ; 5 \mathrm{~V}, P<.001 ; 7.5 \mathrm{~V}, P$ $<.01$; Figure 4).

\section{Molecular Analysis MPG and Penis in Galanin- Treated Rats In Vivo}

Penises and MPGs of sham-, vehicle-, and M1145-treated rats were subjected to polymerase chain reaction analysis to assess differences in expression of structural parameters between treatment groups. In the MPG, no differences in gene expression were seen for neuronal markers nNOS, TH, and $\beta_{3}$-tubulin between vehicle- and M1145-treated rats (Figure 5A). 4 weeks after BCNI, galanin levels remained significantly upregulated in vehicle- and M1145-treated rats $(P<0.001$ vs sham). In the penis, no differences could be seen in expression between vehicle 
A
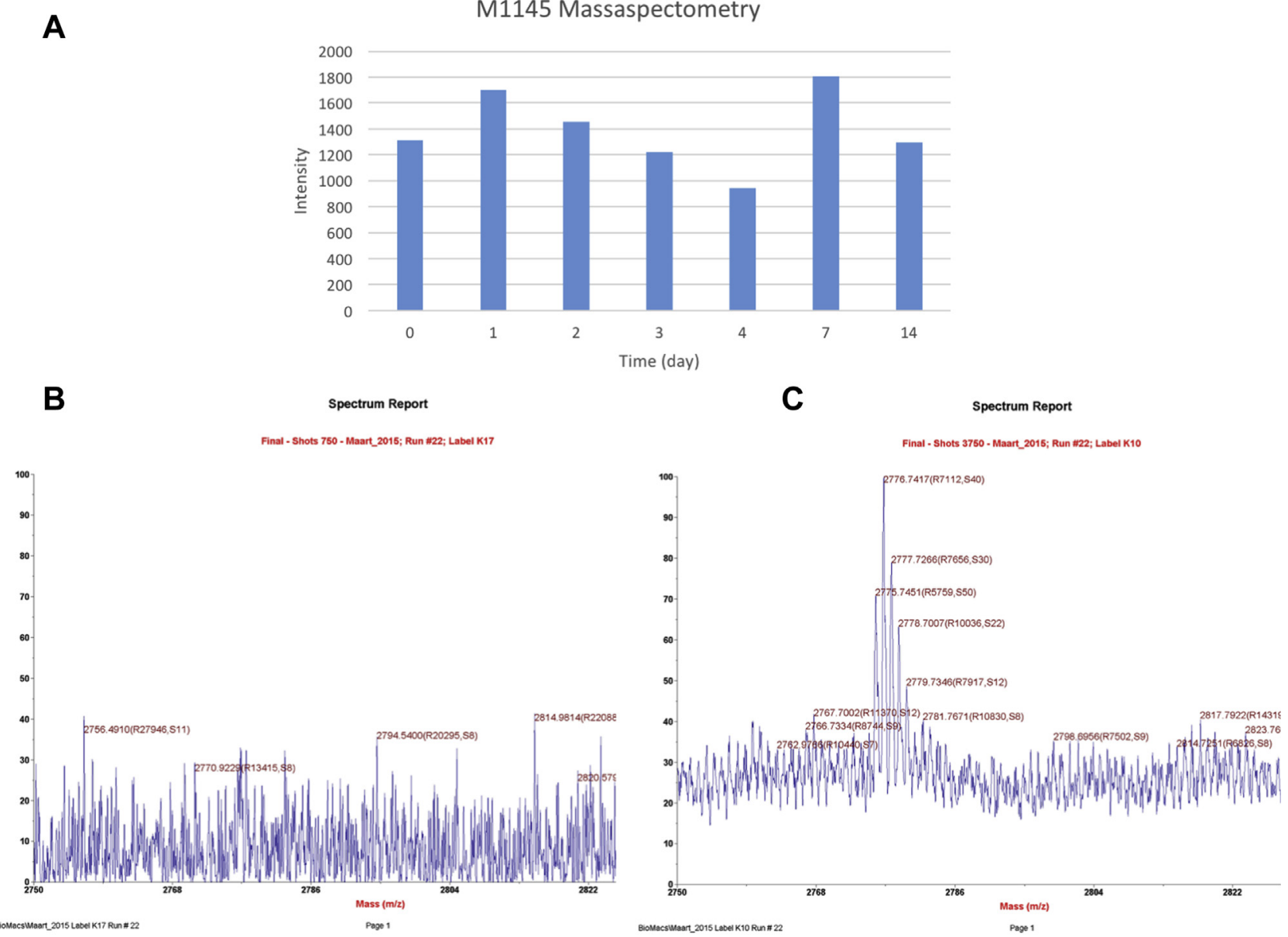

Figure 3. Mass spectrometry of M1145 compound. Panel A shows the intensity of the detected compound remained stable, showing only minimal variance over time. Panels B and C show blood serum samples obtained from M1145-treated rats had a mass spectrometric spectrum and molecular weight consistent with M1145, which was absent in vehicle-treated rats, respectively.

and M1145 for collagens I and III, $\alpha$-smooth muscle actin, TH, and $\mathrm{nNOS}$ (Figure 5B).

\section{Localization Galanin and Its Receptor in Human Neurovascular Bundle}

Slides were selected to contain parasympathetic neuronal ganglion bodies and cavernosal nerve fibers in the periprostatic fat, shown by immunoreactivity to nNOS (Figure 6). For classic chromogenic immunohistochemistry, a staining protocol on adjacent slides was used for galanin receptors and nNOS antibody. All 3 galanin receptors were present in the neuronal cell bodies, although the staining pattern directed against GalR2 was less intense than those against GalR1 and GalR3. The staining pattern in the neuronal cell bodies was identical to that of nNOS on adjacent slides. $\mathrm{CN}$ fibers were positive for nNOS but were not stained by galanin receptor antibodies (Figure 6). Galanin antibody stained the neuronal cell bodies and the staining showed a vesicular pattern. The periganglionic satellite cells surrounding the neuronal bodies were positive for galanin, whereas only minimal background staining was seen in the surrounding periprostatic fat tissue (Figure 6). Definite colocalization was confirmed using immunofluorescent staining, which showed colocalization of galanin and its 3 receptors in the nNOS-positive neuronal cell bodies, providing the nitrergic input to the corpora cavernosa (Figure 7).

\section{DISCUSSION}

This study is a follow-up to a previous study in which we showed that galanin was 180 -fold upregulated in the early phase after CNI and was present in nNOS-positive neurons of the MPG. ${ }^{6}$ In this study we found that inhibition of endogenous galanin resulted in impairment of neurite outgrowth in vitro, which was mediated by GalR2. In a dissociated nerve culture model, we identified that inhibition of neurite outgrowth, however modest, occurred selectively in nitrergic neurons. Addition of galanin agonists in non-nerve-injured MPGs did not result in a significant increase in neurite outgrowth. We also investigated the in vivo potential of galanin administration in enhancing EF recovery after BCNI. We found that systemic administration of M1145, a selective GalR2 agonist, resulted in partial recovery of EF 4 weeks after BCNI compared with vehicle-treated rats. Molecular analysis of the penis and MPG 


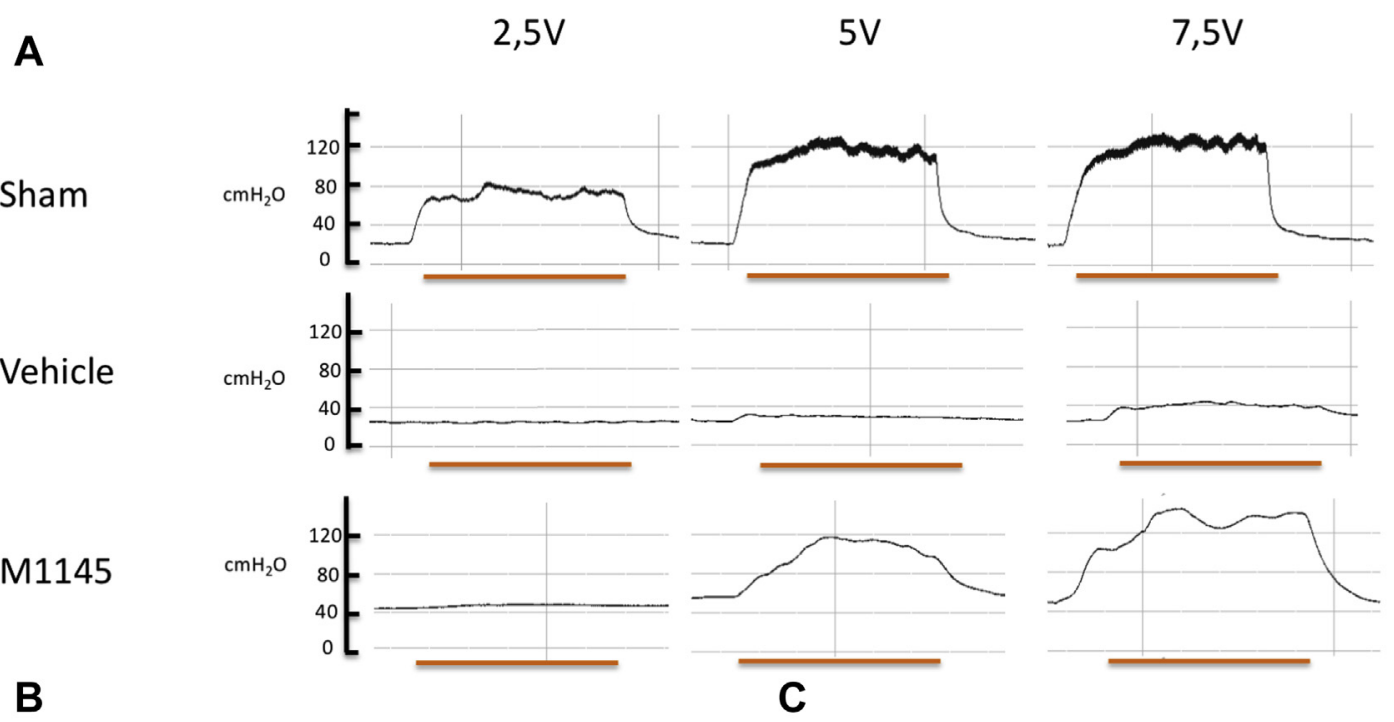

ICP/MAP Mean

ICP/MAP Max
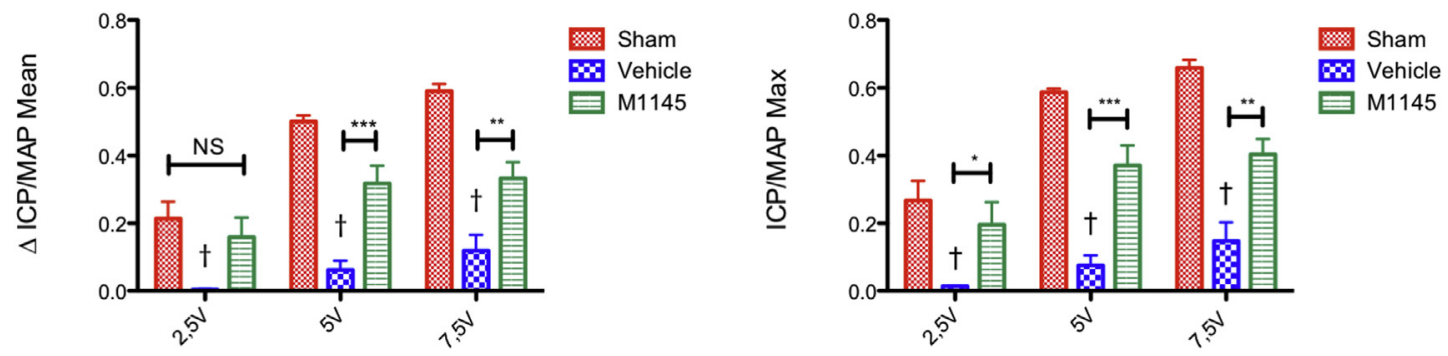

Figure 4. Exogenous galanin agonist administration enhances erectile function recovery in vivo. Panel A shows exemplary traces of erectile response (mean ICP/MAP and maximum ICP/MAP) to electrostimulation of the cavernous nerve in sham-, vehicle-, and M1745treated rats. Horizontal bar below each trace represents duration of stimulation. Panels B and C show mean and maximal erectile responses to electrostimulation, respectively. In rats treated with vehicle, bilateral cavernous nerve injury resulted in significantly impaired erectile responses. In rats treated with M1145, partial recovery of erectile responses to electrostimulation was seen. ${ }^{*} P<0.05$; ${ }^{* *} P<0.01$; ${ }^{* * *} P<0.001\left(\mathrm{n}=10 /\right.$ group); ${ }^{\dagger} P<.001$. ICP $=$ intercavernosal pressure; MAP $=$ mean arterial pressure; $\mathrm{NS}=$ non-significant.

after treatment did not show differences in the expression of structural myogenic and neuronal markers. In additional experiments, we evaluated whether this increase in EF recovery could be explained by a direct vasodilatory action of galanin. However, we did not see a relaxant effect on human corpus cavernosum by pharmacologic modulation of galanin receptors in vitro (Supplementary Figure 1). In addition, we found that galanin and its receptors were present in nitrergic erectile innervation in the human periprostatic neurovascular plexus.

Galanin was previously suggested to be an important mechanism in $\mathrm{CN}$ regeneration and $\mathrm{EF}$ recovery after $\mathrm{CNI}^{6}$ and in this study we have shown that galanin influences the growth of pelvic neurons in vitro. Interestingly, no spectacular increase in neurite outgrowth was achieved when adding galanin agonists to MPGs obtained from rats in a steady state (ie, non-nerve injury; Figure 1). Increasing the number of animals per group might have resulted in significant results because a trend toward increased outgrowth was visible when treating with galanin agonists. In contrast, Girard et $\mathrm{al}^{13}$ reported that explant culture itself results in 20-fold upregulation of galanin. Therefore, exogenous galanin addition might not have shown an additional benefit in a saturated system that was already endogenously upregulated galanin in response to BCNI. Addition of galanin antagonists significantly impaired neurite outgrowth in MPGs that had shifted to a regenerative phenotype by previous BCNI (Figure 1). The neurotrophic effect was mediated by GalR2. This is consistent with previous findings in GalR2 knockout mice showing decreased neurite outgrowth in in vitro DRGs.?

Dissociated nerve culture showed that galanin primarily mediates outgrowth of nitrergic neurons with minimal impact on sympathetic neurons (Figure 2). This has important therapeutic consequences because penile muscle strips from patients after RP show impaired nitrergic relaxation and increased sensitivity to sympathetic contraction. ${ }^{14}$ Hsieh et $\mathrm{al}^{15}$ showed that, after injury to the $\mathrm{CNs}$, regenerated sympathetic fibers seemed to outweigh regenerated parasympathetic fibers. It has been suggested that ablation of a specific neuronal subpopulation by "immunosympathectomy" could be therapeutic in specific neurologic conditions, such as CNI after RP. ${ }^{16,17}$ As our results suggest, we hypothesize that administration of galanin would preferentially stimulate parasympathetic neurons in such way that could counterbalance sympathetic reinnervation after RP and restore $\mathrm{EF}$. 
A Major pelvic ganglion
B3 tubulin

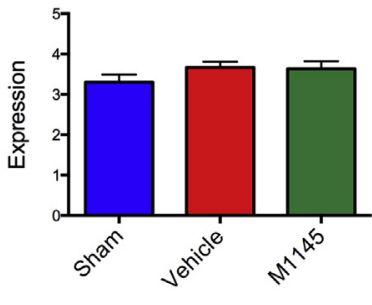

Treatment

\section{B Corpus cavernosum}

aSMA

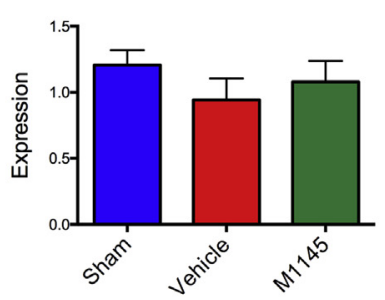

Treatment
TH

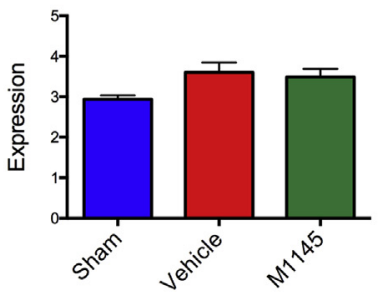

Treatment

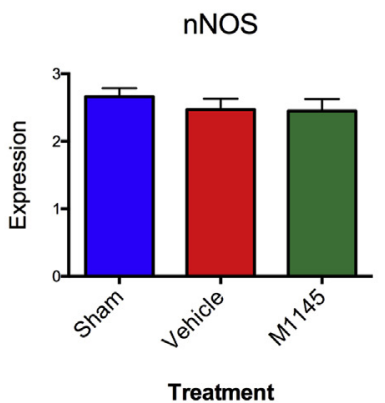

Treatment

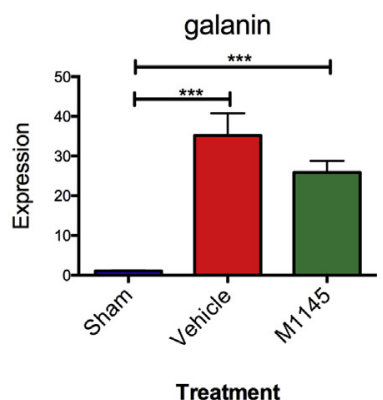

TH

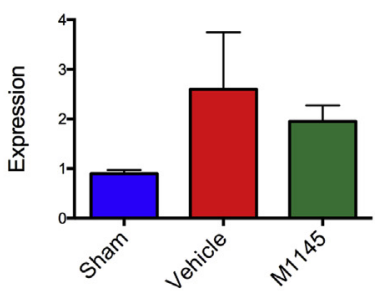

Treatment

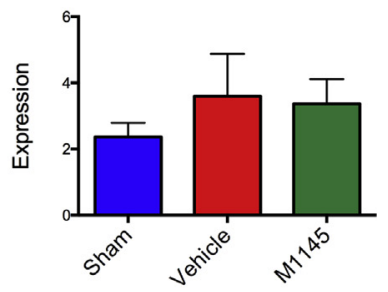

Treatment

Figure 5. Quantitative polymerase chain reaction analysis of major pelvic ganglion and penis in galanin-treated rats in vivo. Panel A shows changes in gene expression in the major pelvic ganglion. No differences in $\mathrm{TH}$, nNOS, and $\beta_{3}$-tubulin were seen between groups. 4 weeks after bilateral cavernous nerve injury, galanin levels remained significantly upregulated in vehicle- and M1145-treated rats. Panel B shows that, in the penis, no differences in gene expression could be seen between vehicle- and M1145-treated rats for collagens I and III, aSMA, TH, and $\mathrm{nNOS}$. ${ }^{* * *} \mathrm{P}<$.0001. aSMA $=\alpha$-smooth muscle actin; Coll $1=$ collagen $\mathrm{l}$; $\mathrm{nNOS}=$ neuronal nitric oxide synthase; $\mathrm{TH}=$ tyrosine hydroxylase.

The neurotrophic effect of galanin in vivo was investigated in the recovery of EF after CNI. Because no data are available on therapeutic doses of galanin in vivo, we recalculated the dosage from a clinical trial with ciliary neurotrophic factor and corrected for molecular weight and normalized for body surface area. ${ }^{12,18} \mathrm{~A}$ strength of our study is that the pharmacokinetic conditions of treatment were thoroughly checked upfront. Stability of the dissolved compound and presence in blood serum were confirmed with mass spectrometry (Figure 3). The wide overall application of neurotrophic factors has been limited by the method of administration, which has to be local or systemic, but not enteral. ${ }^{19}$ In a pilot study, local administration using an intraperitoneal catheter was unsuccessful because an extensive local inflammatory response to the catheter in sham-operated and treated animals made later functional evaluation technically impossible.
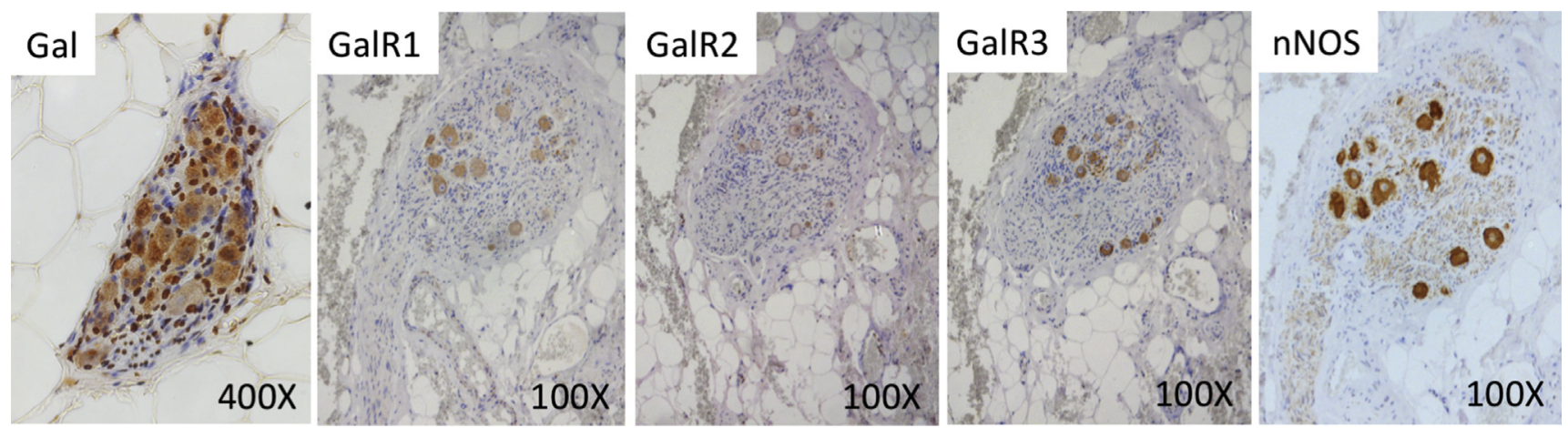

Figure 6. Galanin and its receptors are present in the human neurovascular bundle. Slides were selected to contain nNOS-positive parasympathetic neuronal ganglion bodies and cavernosal nerve fibers in the periprostatic fat, which belong to the human neurovascular bundle responsible for the erectile innervation. All 3 galanin receptors were present in the neuronal cell bodies. The staining pattern in the neuronal cell bodies was identical to that of nNOS on adjacent slides. Gal = galanin; GalR1 = galanin receptor-7; GalR2 = galanin receptor-2; GalR3 = galanin receptor-3; nNOS = neuronal nitric oxide synthase. 

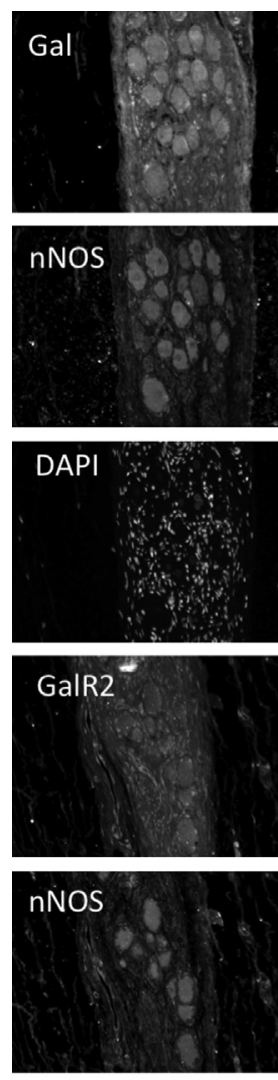

DAPI

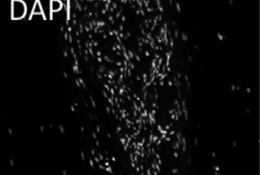

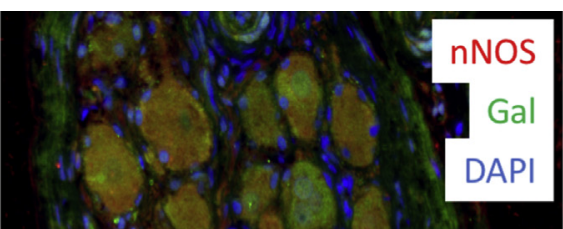
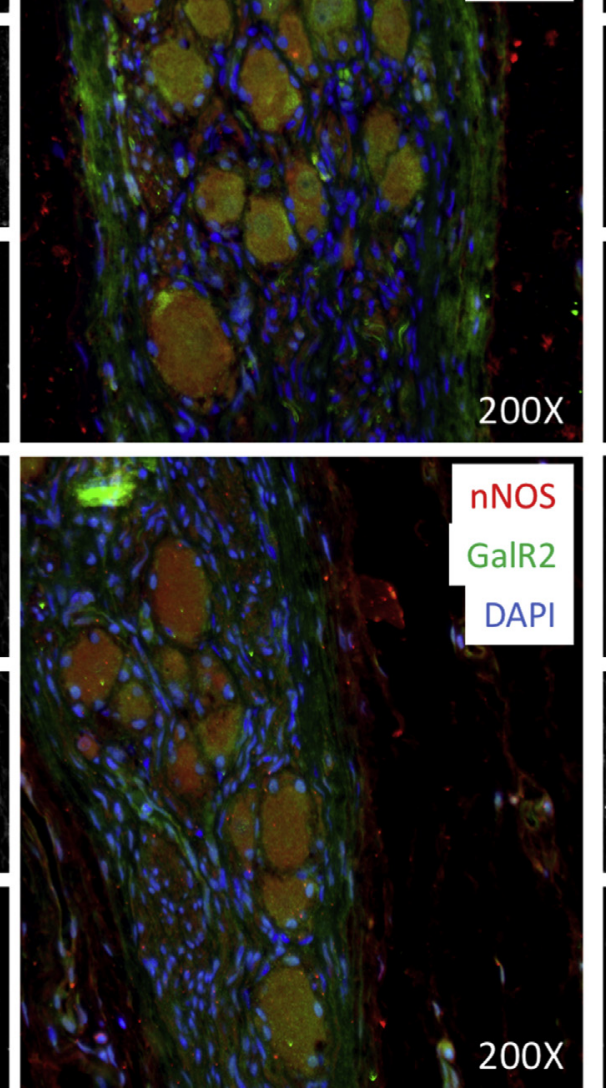
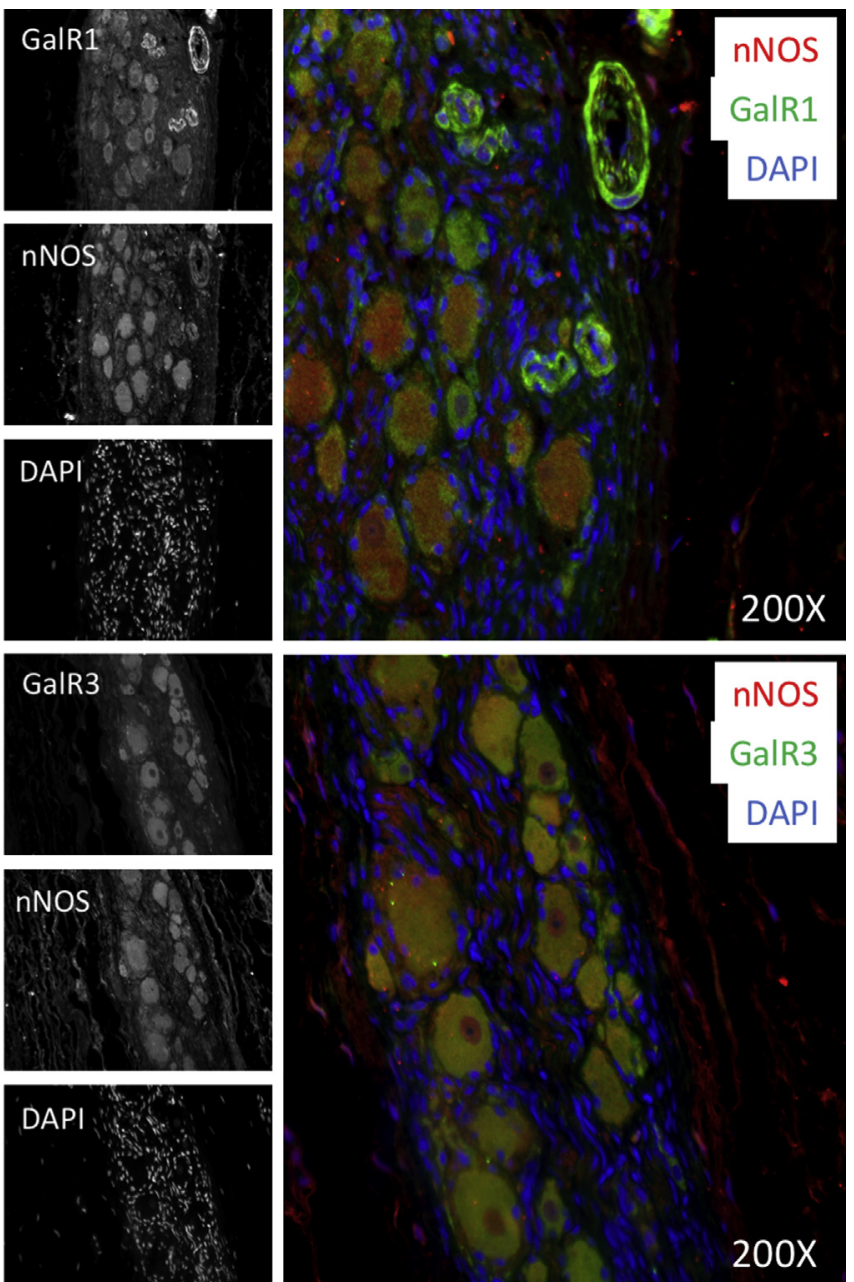

Figure 7. Immunofluorescent staining of galanin and its receptors in the human neurovascular bundle. Immunofluorescent staining shows colocalization of galanin and its 3 receptors (green) in the nNOS-positive neuronal cell bodies (red) providing nitrergic input to the corpora cavernosa. DAPI = 4',6-diamidino-2-phenylindole; Gal = galanin; GalR1 = galanin receptor-7; GalR2 = galanin receptor-2; GalR3 = galanin receptor-3; nNOS = neuronal nitric oxide synthase.

We found that systemic administration of a selective GalR2 agonist, M1145, partly restored EF after BCNI (Figure 4). Surprisingly, no differences between active and vehicle-treated rats could be found when looking at changes in expression of structural markers in the MPG and penis (Figure 4). A possible explanation is that changes in expression at the mRNA level are temporary. We previously reported that endogenous upregulation of galanin was an early event occurring the 1 st few days after injury. ${ }^{6}$ Despite the absence of differences in expression on polymerase chain reaction analysis, arguments still favor neurotropic action as a mechanism for the observed increase in EF recovery. In the 1st part of our study, we found a mechanism of action because galanin mediate neurite outgrowth in vitro (Figures 1 and 2). Furthermore, in other studies, exogenous administration of galanin decreased neuropathic pain and increased neuroregeneration after sciatic nerve-pinch injury ${ }^{20}$ and promoted sciatic nerve recovery in diabetic rats. ${ }^{21}$ Studies using a galanin-overexpressing transgenic mouse line have shown that the total number of DRG neurons lost after axotomy is less than in wild-type controls, indicating a rescue effect of the high levels of galanin. ${ }^{22}$ Interestingly, endogenous upregulation of galanin in the MPG persisted up to 4 weeks after injury, although at lower levels than observed in the early response to injury as described in our previous study (Figure 5). After BCNI, pelvic neurons assume a regenerative phenotype resulting in the upregulation of a number of neuropeptides. In relative proportion, the upregulation of galanin is at least 20 times higher than those of classic neurotrophic factors such as brain-derived neurotropic factor, nerve growth factor, and others. ${ }^{5}$ As discussed previously, the question remains how this endogenous upregulation influences the potential of further stimulation by additional exogenous administration.

Because no additional surgery was performed for pump explantation before in vivo testing, no washout period occurred. Therefore, the increase in erectile response to electrostimulation might be explained in part by a direct effect on erections. Intracavernous injection of galanin and galantide (M15, a non-specific 
galanin receptor antagonist) provoked erections when injected in the corpus cavernosum of cats. ${ }^{23}$ However, we could not see a direct vasodilatory effect of galanin agonists or antagonists in vitro on human (dose response; Figure S1) or rat (1 dose; Figure S2A) penile muscle strips. Using electrical field stimulation, we investigated whether galanin could act as a modulator of the release of gaseous neurotransmitters, such as NO. However, we did not see differences in non-noradrenergic, non-cholinergic transmitter relaxation of phenylephrine pre-contracted strips with or with galanin agonists (Figure S2B). The study by Bivalacqua et $\mathrm{al}^{23}$ was an in vivo study, whereas in our study an in vitro protocol on isolated human cavernosal strips was used. It cannot be excluded that the galanin pathway has a pro-erectile effect by a central action on the central nervous system, more specifically at the level of the spinal cord. In analogy, galanin pathways in the spinal cord are involved in the function of different pelvic organs. Using intrathecal pharmacologic administration, galanin has been shown to be a facilitator of ejaculation ${ }^{24}$ and to influence bladder function in rats. ${ }^{25}$ Furthermore, Keast ${ }^{26}$ described the presence of galanin in adrenergic and parasympathetic preganglionic axons in the MPG.

We found that galanin and its receptors are present in the human periprostatic neurovascular plexus that provides erectile innervation to the penis (Figures 6 and 7). Immuno-electronic studies have shown that galanin is packed in large dense-core vesicles in the Golgi complex of DRG neuronal cell bodies. The granular immunohistochemical staining pattern in the cell body directed against galanin is consistent with its storage in vesicles. In axotomized neurons, galanin is transported in anterograde fashion from the neuronal cell body soma and an accumulation can be seen at the distal regenerating nerve ending. ${ }^{27}$ The presence of the 3 receptors for galanin at the same site of the production of its ligand galanin (ie, in the neuronal cell body) suggests that the galanin pathway functions as an autocrine protective mechanism that stimulates neuroprotection and neuroregeneration after CNI. Newborn galanin knockout mice show a 3-fold increased number of apoptotic DRG postnatally, which indicates that galanin exerts direct paracrine effects on survival within the DRG in the early postnatal period. ${ }^{28}$ The important translational value is that exogenous galanin and its pathway could be used as a target to enhance neuroregeneration after nerve injury during nerve-sparing RP. Preferentially, the application would be local, for example, as a slow-release gel on the neurovascular bundle at the end of the nerve-sparing RP procedure. There might be benefit in a combination of neurotrophic factors, as illustrated by a study that showed accelerated return to potency with dehydrated amnion membrane application. ${ }^{29}$

Our study has some limitations. Western blot analysis was not performed to assess changes in protein expression in nerve culture in vitro. For in vivo testing, no washout period occurred. Although an increase in EF recovery was seen, no differences in the gene expression of structural markers were detected and protein expression was not performed. Therefore, additional research is needed to provide definitive proof of increased neuroregeneration. Furthermore, no antagonist was used in the in vivo experiment. Relaxation mediated by electrical field stimulation was performed on only rat, and not human, penile tissue. No western blot quantification was performed to assess changes in protein expression in vitro for nerve culture experiments. The inclusion of multiple dosage regimes and addition of additional time points would provide more insight before translation and clinical application and will be considered in future studies.

\section{CONCLUSION}

In this study we showed that galanin has neurotrophic action in vitro on neurons isolated from the MPG. Inhibition of endogenous galanin significantly impaired neurite outgrowth, which primarily occurred in nitrergic neurons. In vivo administration of a selective GalR2 agonist, M1145, resulted in partial recovery of EF after BCNI. Molecular analysis of the penis and MPG after treatment did not show differences in gene expression of structural myogenic and neuronal markers. A direct vasodilatory effect is unlikely, because galanin agonist and antagonist did not relax the rat or human corpus cavernosum. Within the human periprostatic neurovascular plexus, galanin and its receptors are present in the neuronal body of nitrergic nerves that provide erectile innervation to the penis and their localization suggests an autocrine protective mechanism. In conclusion, our findings identify the galanin pathway as an important endogenous mechanism in neuroregeneration after CNI. Further research is needed to evaluate whether this already profound endogenous activity might limit additional therapeutic modulation with exogenous galanin as a strategy to enhance EF recovery after RP.

\section{ACKNOWLEDGMENTS}

Special thanks go to Derya Ayaz for her help optimizing the dissociated neuron culture protocol. We also thank Prof Etienne Waelkens and Sandy Vandoninck for their help performing the mass spectrometry experiments and Maarten Falter, Ine Bollen, Lennert Minten, and Willemien Marnef for the human penile muscle myographic studies. We also thank Xiaopu Liu, Godelieve Verbist, Rita Van Bree, Katrien Luyten, and Petra Stevens for the excellent technical and administrative support.

Corresponding Author: Maarten Albersen, MD, PhD, Herestraat 49, 3000 Leuven, Belgium. Tel: 32-16-34-69-30; Fax: 32 16-34-69-31; E-mail: Maarten.albersen@uzleuven.be

Conflicts of Interest: The authors report no conflicts of interest.

Funding: Research grant from the European Society for Sexual Medicine. 


\section{STATEMENT OF AUTHORSHIP}

\section{Category 1}

(a) Conception and Design

Emmanuel Weyne; Trinity J. Bivalacqua; Maarten Albersen

(b) Acquisition of Data

Emmanuel Weyne; Johanna L. Hannan; Thomas Gevaert; Yodi Soebadi; Hotaka Matsui; Fabio Castiglione

(c) Analysis and Interpretation of Data Emmanuel Weyne; Koenraad van Renterghem; Frank Van der Aa; Trinity J. Bivalacqua; Maarten Albersen

\section{Category 2}

(a) Drafting the Article Emmanuel Weyne; Johanna L. Hannan; Maarten Albersen

(b) Revising It for Intellectual Content Thomas Gevaert; Hotaka Matsui; Fabio Castiglione; Koenraad van Renterghem; Dirk De Ridder; Frank Van der Aa; Trinity J. Bivalacqua

\section{Category 3}

(a) Final Approval of the Completed Article Emmanuel Weyne; Johanna L. Hannan; Thomas Gevaert; Yodi Soebadi; Hotaka Matsui; Fabio Castiglione; Koenraad van Renterghem; Dirk De Ridder; Frank Van der Aa; Trinity J. Bivalacqua; Maarten Albersen

\section{REFERENCES}

1. Weyne E, Mulhall J, Albersen M. Molecular pathophysiology of cavernous nerve injury and identification of strategies for nerve function recovery after radical prostatectomy. Curr Drug Targets 2015;16:459-473.

2. Boorjian SAS, Eastham JAJ, Graefen MM, et al. A critical analysis of the long-term impact of radical prostatectomy on cancer control and function outcomes. Eur Urol 2012; 61:664-675.

3. Weyne E, Castiglione F, Van der Aa F, et al. Landmarks in erectile function recovery after radical prostatectomy. Nat Rev Urol 2015;12:289-297.

4. Fode $M$, Ohl DA, Ralph $D$, et al. Penile rehabilitation after radical prostatectomy: what the evidence really says. BJU Int 2013;112:998-1008.

5. Hannan JL, Albersen M, Stopak BL, et al. Temporal changes in neurotrophic factors and neurite outgrowth in the major pelvic ganglion following cavernous nerve injury. J Neurosci Res 2015;93:954-963.

6. Weyne E, Albersen M, Hannan JL, et al. Increased expression of the neuroregenerative peptide galanin in the major pelvic ganglion following cavernous nerve injury. J Sex Med 2014; 11:1685-1693.

7. Melander T, Hökfelt T, Rökaeus A. Distribution of galaninlike immunoreactivity in the rat central nervous system. J Comp Neurol 1986;248:475-517.

8. Zhang $X, X u$ Z-Q, Shi T-J, et al. Regulation of expression of galanin and galanin receptors in dorsal root ganglia and spinal cord after axotomy and inflammation. Ann N Y Acad Sci 1998;863:402-413.
9. Hobson SA, Holmes FE, Kerr N. Mice deficient for galanin receptor 2 have decreased neurite outgrowth from adult sensory neurons and impaired pain-like behaviour. J Neurochem 2006;99:1000-1010.

10. Hobson S-A, Vanderplank PA, Pope RJP, et al. Galanin stimulates neurite outgrowth from sensory neurons by inhibition of Cdc42 and Rho GTPases and activation of cofilin. J Neurochem 2013;127:199-208.

11. Hannan JL, Matsui H, Sopko NA, et al. Caspase-3 dependent nitrergic neuronal apoptosis following cavernous nerve injury is mediated via RhoA and ROCK activation in major pelvic ganglion. Sci Rep 2016;6:29416.

12. Group ACTS. A double-blind placebo-controlled clinical trial of subcutaneous recombinant human ciliary neurotrophic factor (rHCNTF) in amyotrophic lateral sclerosis. Neurology 1996; 46:1244-1249.

13. Girard BM, Galli JR, Vizzard MA, et al. Galanin expression in the mouse major pelvic ganglia during explant culture and following cavernous nerve transection. J Mol Neurosci 2012; 48:713-720.

14. Martínez-Salamanca Jl, La Fuente JM, Fernández A, et al. Nitrergic function is lost but endothelial function is preserved in the corpus cavernosum and penile resistance arteries of men after radical prostatectomy. J Sex Med 2015;12:590-599.

15. Hsieh PS, Bochinski DJ, Lin GT, et al. The effect of vascular endothelial growth factor and brain-derived neurotrophic factor on cavernosal nerve regeneration in a nerve-crush rat model. BJU Int 2003;92:470-475.

16. Weyne E, Bivalacqua TJ, Albersen M. Immunosympathectomy for preservation of erectile function following cavernous nerve injury. Eur Urol 2015;67:727-728.

17. Cattaneo A. Immunosympathectomy as the first phenotypic knockout with antibodies. PNAS 2013;110:4877-4885.

18. Reagan-Shaw S, Nihal M, Ahmad N. Dose translation from animal to human studies revisited. FASEB J 2008; 22:659-661.

19. Thoenen $H$, Sendtner M. Neurotrophins: from enthusiastic expectations through sobering experiences to rational therapeutic approaches. Nat Neurosci 2002;5:1046-1050.

20. Xu X, Yang X, Zhang P, et al. Effects of exogenous galanin on neuropathic pain state and change of galanin and its receptors in DRG and SDH after sciatic nerve-pinch injury in rat. PLoS One 2012;7:e37621.

21. Xu X-F, Zhang D-D, Liao J-C, et al. Galanin and its receptor system promote the repair of injured sciatic nerves in diabetic rats. Neural Regen Res 2016;11:1517-1526.

22. Holmberg K, Kuteeva E, Brumovsky P, et al. Generation and phenotypic characterization of a galanin overexpressing mouse. Neuroscience 2005;133:59-77.

23. Bivalacqua TJ, Champion HC, Purohit SK, et al. Nitric oxidemediated erectile effects of galantide but not galanin in vivo. Nitric Oxide 2000;4:94-102.

24. Kozyrev N, Coolen LM. Activation of galanin and cholecystokinin receptors in the lumbosacral spinal cord is 
required for ejaculation in male rats. Eur J Neurosci 2017; 45:846-858.

25. Honda M, Yoshimura N, Inoue S, et al. Inhibitory role of the spinal galanin system in the control of micturition. Urology 2013;82:1188; e9-e13.

26. Keast JR. Neuropeptide-containing axon terminals in the male rat major pelvic ganglion are primarily of sacral origin. J Auton Nerv Syst 1994;47:151-158.

27. Shadiack AM, Zigmond RE. Galanin induced in sympathetic neurons after axotomy is anterogradely transported toward regenerating nerve endings. Neuropeptides 1998;32:257-264.
28. Holmes FE, Mahoney S, King Von R, et al. Targeted disruption of the galanin gene reduces the number of sensory neurons and their regenerative capacity. PNAS 2000;97:11563-11568.

29. Ogaya-Pinies G, Palayapalam-Ganapathi H, Rogers $T$, et al. Can dehydrated human amnion/chorion membrane accelerate the return to potency after a nerve-sparing robotic-assisted radical prostatectomy? Propensity score-matched analysis. J Robot Surg 2017;,182:2232-2239.

\section{SUPPLEMENTARY DATA}

Supplementary data related to this article can be found at https://doi.org/10.1016/j.jsxm.2018.02.013. 